\title{
Research on the Conversion Process Model and Dynamic Image Based on Analytic Hierarchy Method and Matrix Theory
}

\author{
Lihua Yang \\ Dongying Vocational College, Dongying, China \\ lihua_yang1@163.com
}

Keywords: Sort numeric vector; Modeldecision coefficient; Dynamic image; Process model; Analytic hierarchy process

\begin{abstract}
Faced with the rapid development of current economic globalization and internationalization, the application of English language attract more and more attention. English is not simply used in daily communication now, more is applied to business negotiations and deeplevel communication and cooperation, so we need pay attention to the English words and their extended meaning. This paper from the basic level perspective, using the analytic hierarchy process from the external and internal factors two aspects to analyse the English words and concepts such as dynamic image. Also constructed English words conversion process model from the two aspects, one is microscopic model, another is macro network model. This helps us to understand the dynamic image of English nouns, to flexiblely use the word conversion process, which can enrich the meaning of the words, and lay a solid foundation for the integration of society and international.
\end{abstract}

\section{Introduction}

With the speed up of internationalization process and the rapid development of economic globalization and information technology, it constantly force us to conform with international, to adapt to the fierce international competition. And this inevitably requires people to pay more attention to English, which not only concerned with daily communication, but also to achieve the deep-level communication, business application and negotiation. so for the English language will pay more attention to details. But at this stage, English linguistics is developing more rapidly, especially for dynamic images of English nouns and words conversion, which are frequently used in English daily communications and exchanges. Aut also the development into specific use form, combined with the context, carry out semantic and grammar fusion, this also caused constant attention by all sectors of the community, also determines its important position in the linguistic circles[1]. This paper from the basic levels to analyse the dynamic image of English nouns, using the analytic hierarchy process from the external and internal factors two aspects, also constructe English words conversion process model. It can provide the basis for us to carry out words conversion and the extension of word meaning, which have certain realistic directive significance.

\section{The literature theory and research method}

Some scholars say that the using of word is the the word collocations people often used for daily needs. English words are not only for the extension and expansion of existing meaning, but also generate new words. With the development of modern society, people' English language experience are continuously enriched, which will creat more meanings, even gradually extend to the polysemous status. And we need combine with the current context, grammar and meaning, to make the language and English words have meaning. Some scholars think that the analysis of semantic must adhere to the " phrases " and " open choice " two basic principles. The phrase principle is to use many existing lexical chunks that don't need oneself to combine directly applied to language statements[2]. But open choice principle need proper and reasonable selection of vocabulary and grammar, starting from zero for statement establishment. There are also scholars from the lexical approach point of view, language is usually contains lexical phrase is prefabricated language block, rather than the general form of vocabulary and grammar[3]. Of course we can research the program characteristics of language and also have language analysis function. The word block of English language is not 
their combination, that is normally present in the human brain potential memory, at the same time when we become more familiar with our English learning materials, the word block amount also will increase.

TABLE I. THE CONTENT AND MEANING OF HIERARCHY ANALYSIS

\begin{tabular}{|c|l|}
\hline $\begin{array}{c}\text { Score } \\
\text { points }\end{array}$ & \multicolumn{1}{c|}{ Basic content } \\
\hline 1 & Two factors compared, have the same importance \\
\hline 3 & Two factors compared, one index factor is slightly important than the other \\
\hline 5 & Two factors compared, one index factor is obviously important than the other \\
\hline 7 & Two factors compared, one index factor is strongly important than the other \\
\hline 9 & Two factors compared, one index factor is extremely important than the other \\
\hline 2,4, & Take the intermediate fraction of the two adjacent scoring segment \\
\hline
\end{tabular}

This paper use the analytical hierarchy process for data processing, first of all is the judge of matrix elements $b_{i j}$.According to the related factors in dynamic image of English nouns and word conversion process models $D_{1}, D_{2} \ldots D_{n}$, namely analyse the influencing factors of these models, after calculating, finally get the model state influence degree of the final target $P$ [4].

$$
\begin{gathered}
D_{i}: D_{j} \Rightarrow b_{i j} \\
B=\left(b_{i j}\right)_{n \times n}, b_{i j}>0, b_{j i}=\frac{1}{b_{i j}}
\end{gathered}
$$

Among them, through the formula of $B$ to identify the value of ${ }^{D_{1}}, D_{2} \ldots D_{n}$, and the weight vector value of target $P$.

Secondly, get the common characteristics of aircraft through pattern data[5]:

$$
M(b=1) \Rightarrow \theta_{1}, \theta_{2}, \cdots \theta_{n}
$$

Then, through combination get the ranking value of the impact factors:

$$
\boldsymbol{b}_{i j}=\theta_{i} / \theta_{j}
$$

According to the numerical vector data computation and comparison, the calculation formula is as follows[6]:

$$
b_{i j} \cdot b_{j k}=b_{i k}, i, j, k=1,2, \ldots, m
$$

So we can write the vector matrix B consistent with the model, namely[7]:

$$
B=\left[\begin{array}{cccc}
\frac{\theta_{1}}{\theta_{1}} & \frac{\theta_{1}}{\theta_{2}} & \cdots & \frac{\theta_{1}}{\theta_{n}} \\
\frac{\theta_{2}}{\theta_{1}} & \frac{\theta_{2}}{\theta_{2}} & \cdots & \frac{\theta_{2}}{\theta_{n}} \\
\cdots \cdots & & \\
\frac{\theta_{n}}{\theta_{1}} & \frac{\theta_{n}}{\theta_{2}} & \cdots & \frac{\theta_{n}}{\theta_{n}}
\end{array}\right]
$$

\begin{tabular}{|c|c|c|c|}
\hline $\begin{array}{l}\text { Influence } \\
\text { index }\end{array}$ & $\begin{array}{l}\text { The weight coefficient of } \\
\text { index }\end{array}$ & Level two indexes of model & $\begin{array}{c}\text { Model decision } \\
\text { coefficient }\end{array}$ \\
\hline \multirow{5}{*}{ External factors } & \multirow{5}{*}{0.8952} & The meaning of English nouns & 0.9638 \\
\hline & & $\begin{array}{l}\text { The subjective meaning of English } \\
\text { nouns }\end{array}$ & 0.9723 \\
\hline & & The emotion contains in the meaning & 0.9739 \\
\hline & & Static images of meaning & 0.9528 \\
\hline & & Dynamic images of meaning & 0.9826 \\
\hline \multirow{4}{*}{ Internal factors } & \multirow{4}{*}{0.9048} & Meaning of noun & 0.9854 \\
\hline & & Similar meaning of words & 0.9268 \\
\hline & & The conversion background of meaning & 0.9737 \\
\hline & & The conversion conditions of meaning & 0.9621 \\
\hline
\end{tabular}

Based on the contracted dynamic image of English nouns and word conversion process model, according to the factor influencing degree and expert scoring form reachs the weight of each index value, shown in Table 2.

TABLE II. STATISTICS OF DYNAMIC IMAGE OF ENGLISH NOUNS AND WORDS CONVERSION PROCESS MODEL INDEX 
From the data statistics of dynamic image of English nouns and conversion process model we can know, the factors that influence dynamic image and word conversion are external and internal factors, and for external factors, also contains many level two indexes. External factors include: the meaning of English nouns, the subjective meaning of English nouns, the emotion contains in the meaning, static images of meaning, dynamic images of meaning[8]. Among these level two indicators, the largest index is the dynamic images of meaning, decision coefficient is 0.9826 , followed by the emotion contains in the meaning, decision coefficient is 0.9739 . The minimum influence is the static image, decision coefficient is 0.9528. Internal factors not only have level two indexes like meaning of nouns, similar meaning of words, the conversion background of meaning, the conversion conditions of meaning, which, the meaning of nouns is the maximum impact, decision coefficient is 0.9854 , followed by the conversion background of meaning, decision coefficient is 0.9737 , the minimal impact is similar meaning of words, decision coefficient is 0.9268. Compared with internal factors, the external factor plays an important role, the weight coefficient index is 0.9048 .

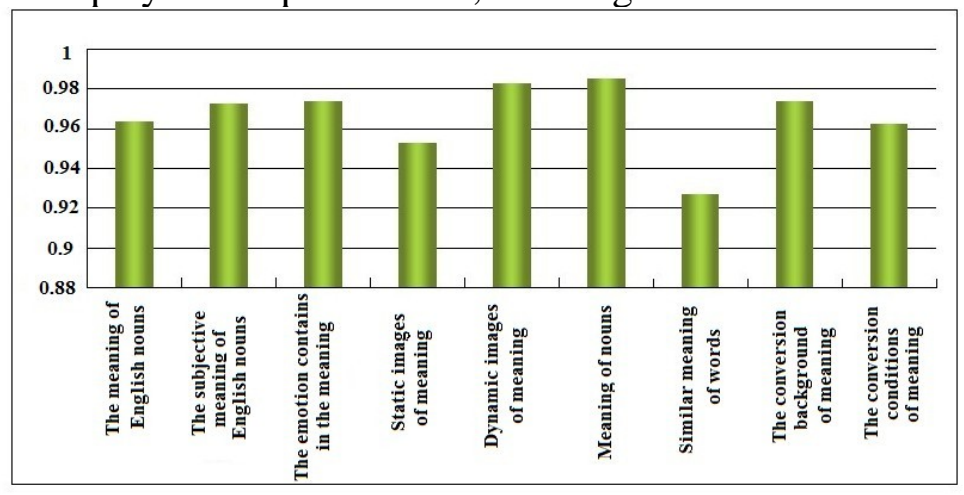

Figure 1. Model two level effects of index decision coefficient

From Figure 1 and Figure 2 we can know, there are some differences exist between the decision coefficients of level two impact index, which also reflects the the influence degree of different factors for dynamic images of English nouns and conversion process are not different. But for the external factors, level two impacts of various index decision coefficient substantially differ not quite, but for internal factors, the decision coefficient of similar meaning of words is very small, which is the smallest in the internal and external factors, namely have the smallest impact on dynamic images of English nouns and words conversion process. It also explains is very important for the selection of index factors, also plays an important role in the dynamic images of English nouns and words conversion process[9].

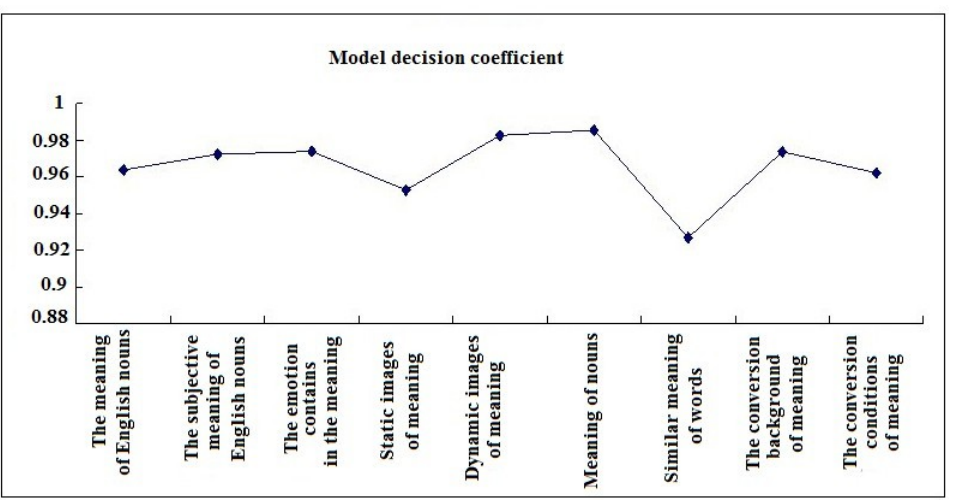

Fig.2 The trend graph of factors decision coefficient of dynamic image and conversion process model

\section{Analysis on English word conversion process model}

Due to people have organized and order on the cognition of things, also organized and law on English words conversion process. English words conversion mode mainly includes the following kinds[10,11]:

(1)English words conversion micro structure pattern

The micro pattern of English words, also known as the change mode of English word conversion chain nodes, namely a complex process from the original meaning to the extend meaning. Based on 
the semantics to do cognitive research and analysis, get the conversion of meaning is closely related to human's cognitive progress, it is the results of human cognitive category and concept. This shows that, English word conversion process model is constructed by means of human cognitive and cultural models. Through the concept conversion of the basic meaning transform the words to get the cognitive acquisition. Along with the improve of human cognitive and cultural level, the original meaning of English words have new understanding and development, gradually formed the words conversion. The micro pattern structure of English word conversion process are shown in Figure 3.

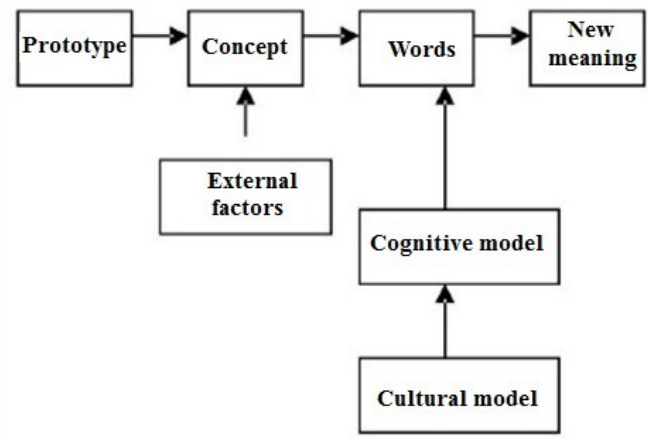

Figure3.The micro pattern chart of English words conversion process

The macro network pattern of English words conversion

Each English word includes a basic meanings and a artistic conception. According to the meaning prototype theory's explanation, this phenomenon is the word prototype. On this basis, the dynamic image understanding and transforming words to form a new word or part of speech, namely the extended meaning. In the new environment, the extended meaning has gained new understanding and the category of word conversion extension to continue. The macro network conversion pattern structure diagram is shown in Figure 4.

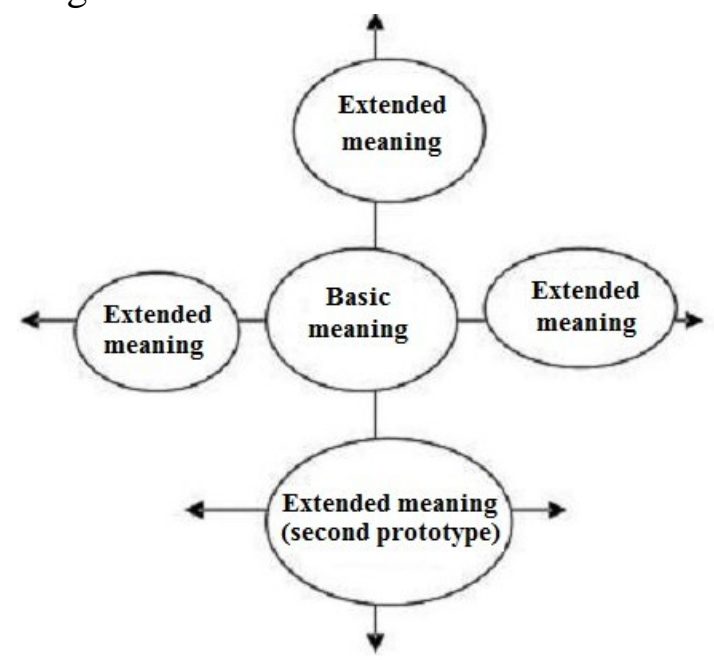

Figure4.The macro network conversion pattern chart of English words

Figure 4 shows the English word conversion process ways, conversion and conversion path and semantic distribution, this also reflects the direction and trend of English word conversion process. English nouns not only has its own basic meaning, but also expands the basis to develop a few direction for several extended meaning, at the same time some extended meaning and can be used as a second prototype, thus further develop many extended meanings, which will makes the English words have more abundant connotation.

\section{Conclusion}

Currently, the English language is in the period of rapid development, we need to pay enough attention to English linguistics, English words are not only the extension and expansion of existing meaning, but also to generate new words. With the development of modern society, people's English language experience are continuously enriched, which will create more meanings, even gradually extend to the polysemous status. And we need to combine with the current context, grammar and 
meaning, to make the language and English words have meaning. Especially in the period when linguistics have received ample attention, we need to keep learning, master dynamic image of English nouns and words conversion process mode, through the microscopic and macroscopic network model for word conversion process, so as to constantly enrich and expand the extended meaning, and continuously enrich the connotation of words. For our better English word learning, mastery of English word usage, make contribute to achieve international standards.

\section{References}

[1] Feng Lingyu.The significance of core sememe in syntactic category discriminant .Studies in Language and Linguistics,2008(1):118-121

[2] Hu Yushu.Modern Chinese (recast ).Shanghai:Shanghai Education Press,2011:113-114

[3] Bai Xiehong.Formation of English imitation words and their translation.China Translation,2011(05):51-52

[4] Bo Bing,Zhao Dexin.A handbook of English grammar.Beijing:The Commercial Press,2011:214-215

[5] Liao Guangrong,Xiao Yunnan. Cognitive research and acquisition of the word meaning evolution of economic and trade English.Journal of Xi'an International Studies University,2009(3):41

[6] Liao Guangrong.Research on the model of polysemy.Foreign Language Teaching,2008(3):12

[7] Liao Guangrong.Research on polysemic category prototype fission, subcategorization and related problems.Foreign Language and it's Teaching,2009(10):30-31

[8] Huang Borong,Liao Xudong.Modern Chinese.Beijing:Higher Education Press,2011:105-107

[9] Li Baojia.Theoretical linguistics -- double spirit of Humanities and Science.Nanjing:Jiangsu Ancient books Press,2011:94-95

[10] Liu Shushun.Theory of modern Chinese.Beijing:Higher Education Press,2010:189-190

[11] Liu Shun.The study of modern Chinese Nouns.Shanghai:Phaidon Press,2008:85-86 